\title{
Compensation of Magnetic Disturbances Improves Inertial and Magnetic Sensing of Human Body Segment Orientation
}

\author{
Daniel Roetenberg, Henk J. Luinge, Chris T. M. Baten, and Peter H. Veltink, Member, IEEE
}

\begin{abstract}
This paper describes a complementary Kalman filter design to estimate orientation of human body segments by fusing gyroscope, accelerometer, and magnetometer signals from miniature sensors. Ferromagnetic materials or other magnetic fields near the sensor module disturb the local earth magnetic field and, therefore, the orientation estimation, which impedes many (ambulatory) applications. In the filter, the gyroscope bias error, orientation error, and magnetic disturbance error are estimated. The filter was tested under quasi-static and dynamic conditions with ferromagnetic materials close to the sensor module. The quasi-static experiments implied static positions and rotations around the three axes. In the dynamic experiments, three-dimensional rotations were performed near a metal tool case. The orientation estimated by the filter was compared with the orientation obtained with an optical reference system Vicon. Results show accurate and drift-free orientation estimates. The compensation results in a significant difference $(p<0.01)$ between the orientation estimates with compensation of magnetic disturbances in comparison to no compensation or only gyroscopes. The average static error was $1.4^{\circ}$ (standard deviation 0.4 ) in the magnetically disturbed experiments. The dynamic error was $2.6^{\circ}$ root means square.
\end{abstract}

Index Terms-Accelerometer, gyroscope, Kalman filter, magnetic disturbance, magnetometer, orientation, sensor fusion.

\section{NOMENCLATURE}

$G_{\mathbf{X}} \quad$ Vector in global reference frame.

$S_{\mathbf{X}} \quad$ Vector in sensor reference frame.

$\hat{\mathbf{x}} \quad$ Estimate.

$\mathbf{x}^{-} \quad$ A priori state estimate using signal model.

$\mathbf{x}^{+} \quad$ A posteriori state estimate after correction by Kalman filter.

\section{INTRODUCTION}

$\mathbf{T}$ HE USE of miniature inertial sensors has become a common practice in ambulatory human movement analysis. Micromachined gyroscopes and accelerometers are used in several applications which include monitoring of activities of daily living [1]-[3], assessment of internal mechanical working load in ergonomics studies [4]-[7], measurement of neurological

Manuscript received April 15, 2004; revised September 30, 2004; accepted January 17, 2005. This work was supported by the Dutch Ministry of Economic Affairs under the AmbuLab project.

D. Roetenberg and P. H. Veltink are with the Biomedical Technology Institute, University of Twente, 7500 AE Enschede, The Netherlands (e-mail: D.Roetenberg@utwente.nl).

H. J. Luinge is with Xsens Motion Technologies, 7500 AM Enschede, The Netherlands.

C. T. M. Baten is with the Roessingh Research and Development, $7500 \mathrm{AH}$ Enschede, The Netherlands.

Digital Object Identifier 10.1109/TNSRE.2005.847353 disorders [8]-[11], and mixed and augmented reality [12]-[14]. It should be noted that there are important limitations in the current systems. The inherent drift of the orientation and position estimates limits long-term stable application of these sensors [15]. Typically, angular orientation is determined by integrating the output from the angular rate sensors. Microelectromechanical (MEMS) gyroscopes are accurate for angular velocity measurements but can only be used for a short time to calculate angular orientation. A relatively small offset error due to temperature effects on the gyroscope signal will introduce large integration errors. Linear accelerometers measure the vector sum of acceleration a and gravitational acceleration $\mathbf{g}$ in sensor coordinates. The orientation calculated using the angular rate sensors can be used to express this vector sum in global coordinates.

The gravitational acceleration component $\mathrm{g}$ is in most situations of human movement sensing dominant, thus providing inclination information [16]-[18]. This can be used to correct the drifted orientation estimate from the gyroscopes. The principles for orientation estimation of a moving human body segment by fusing miniature gyroscopes and accelerometers in a Kalman filter have been described by Luinge [19]. The results show accurate drift-free inclination estimation, though heading (or yaw) drift is the impeding factor for full three-dimensional (3-D) orientation measurement in this filter. Since accelerometers cannot detect rotations about the vertical axis, magnetic sensing can be added. The magnetometer is sensitive to the earth's magnetic field and can thus be used to correct drift of the gyroscope about the vertical axis. Bachmann [12] and Foxlin [14] have implemented filters in which accelerometers and magnetometers are used for low-frequency components of the orientation and gyroscopes to measure faster changes in orientation. However, ferromagnetic materials, like iron, or other magnetic materials in the vicinity of the sensor will disturb the local magnetic field and will, therefore, cause large errors in the estimated orientation, especially in the heading direction. Also, other proposed methods to fuse the three different sensors do not take magnetic interferences into account [20]-[22]. In controlled measurement volumes like laboratories for gait or virtual reality, the magnetic field can be characterized a priori. However, in ambulatory applications with unknown materials and magnetic objects in the environment, inaccuracy in orientation estimation is very likely to occur. In this study, the Kalman filter for body segment orientation by Luinge [23] is extended with a magnetometer model which is preventing heading drift and is able to compensate for magnetic disturbances. This paper focuses on the filter design and the effects ferromagnetic materials near the sensor module under quasi-static and dynamic conditions. 


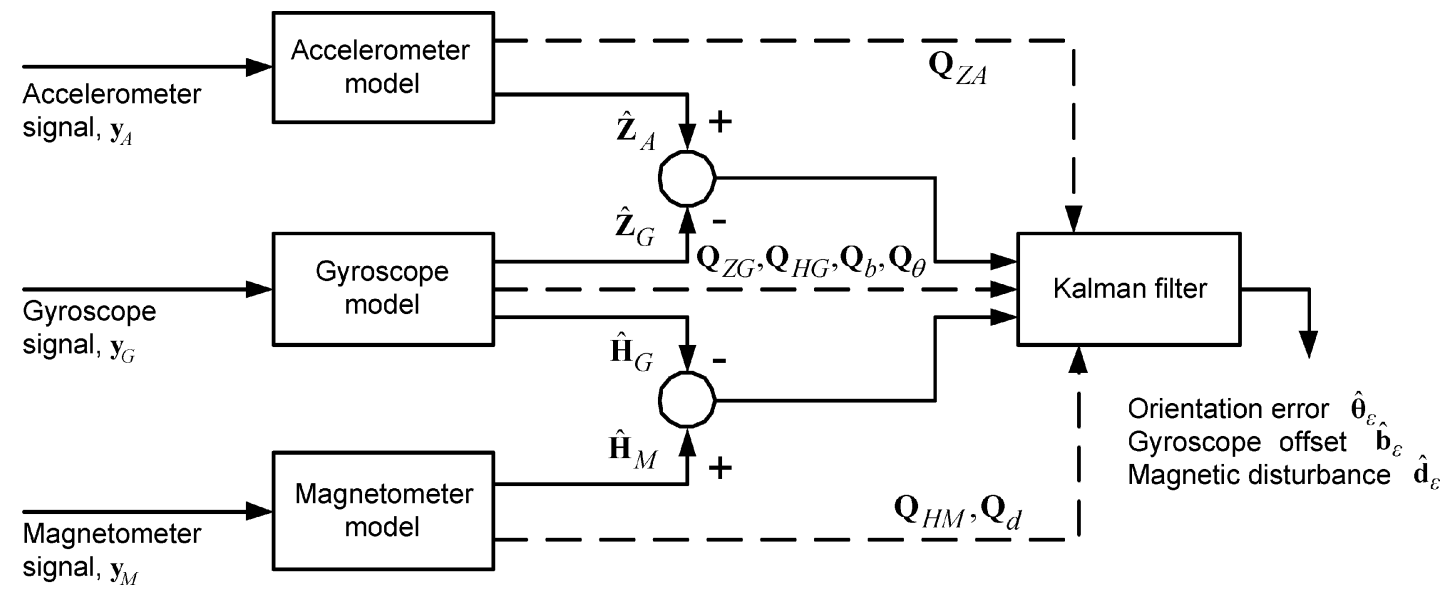

Fig. 1. Fusion of gyroscope $\mathbf{y}_{G}$, accelerometer $\mathbf{y}_{A}$, and magnetometer signals $\mathbf{y}_{M}$ in the error model for combined inertial and magnetic orientation sensing. $\hat{\mathbf{Z}}_{A}$ and $\hat{\mathbf{Z}}_{G}$ are the estimates of inclination by accelerometer and gyroscope, respectively, with $\mathbf{Q}_{Z A}$ and $\mathbf{Q}_{\text {ZG }}$ being the related error covariance matrices. $\hat{\mathbf{H}}_{G}$ and $\hat{\mathbf{H}}_{M}$ are the estimates of the global magnetic field vector by gyroscope and magnetometer, respectively, with $\mathbf{Q}_{H G}$ en $\mathbf{Q}_{H M}$ being the related error covariance matrices. Differences between the sensor estimates in $\mathbf{Z}$ and $\mathbf{H}$ are written as a function of the orientation error $\hat{\boldsymbol{\theta}}_{\varepsilon}$, gyroscope offset error $\hat{\mathbf{b}}_{\varepsilon}$ and magnetic disturbance error $\hat{\mathbf{d}}_{\varepsilon}$ and the related covariance matrices $\mathbf{Q}_{\theta}, \mathbf{Q}_{b}$, and $\mathbf{Q}_{d}$, the estimated errors by the Kalman filter are used to correct the estimated orientation and signal predictions, as illustrated in Fig. 2.

\section{METHOD}

\section{A. Sensor Fusion}

A complementary Kalman filter was designed to estimate orientation by combining the signals of a complete 3-D sensor module including three rate gyroscopes, three accelerometers, and three magnetometers. The structure of the error estimation procedure is shown in Fig. 1. The Kalman filter is useful for combining data from several different indirect and noisy measurements. Thus, while gyroscopes measure orientation by integrating angular velocities and the accelerometer (as an inclinometer) and magnetometer (as a compass) provide a noisy and disturbed but drift-free measurement of orientation, the Kalman filter weights the three sources of information appropriately with knowledge about the signal characteristics based on their models to make the best use of all the data from each of the sensors[14].

From this combination or fusion of sensor signals, information is obtained regarding the offsets of the gyroscopes, accelerometers, and magnetometers, which can be used to recalibrate the sensors in use. The model of the measured gyroscope and acceleration signals is based on the following assumptions [23].

1) A gyroscope measures a 3-D angular velocity plus an offset and white measurement noise in the sensor coordinate frame.

2) The spectrum of the gyroscope offset has a low bandwidth in comparison with the spectrum of the kinematic signals that are to be measured.

3) A 3-D accelerometer measures the sum of acceleration of the movement, gravitational acceleration $(\approx$ $9.8 \mathrm{~ms}^{-2}$ ), and a white noise component, all in the sensor coordinate frame.

4) The acceleration of the body segment in the global system can be described as low-pass filtered white noise.

In this study, the following additional assumptions are used.

1) A 3-D magnetometer measures the earth magnetic field vector plus a white noise component.
2) The magnetic field can be disturbed by nearby ferromagnetic materials or magnetic fields other than the earth magnetic field. The bandwidth of the disturbance is larger than the bandwidth of the gyroscope drift and is dependent of the movement.

The complementary Kalman filter is not based on the model of the process, but on a model of errors. The advantages are that this structure maintains the high dynamic response necessary for attitude state variables and most error processes in the inertial measurement units (IMUs), can be described by linear processes [24]. The four parts of the complementary filter are: the a priori model prediction of the state, the error model, the Kalman filter, and the state correction yielding the a posteriori state estimate (see Fig. 2). A hat on top of symbol denotes an estimate, a minus superscript the a priori estimate that is made using the sensor model and a plus superscript an estimate that is made after correction by the filter.

\section{B. Prediction Model}

The sensor is assumed to be attached to a human body segment that rotates and translates with respect to a global coordinate frame. The strapdown integration algorithm by Bortz [25] was used to calculate the change in orientation from an angular velocity.

The orientation of the sensor with respect to the global coordinate frame $\mathrm{G}$ is expressed with a rotation matrix $\mathbf{R}$, containing the three unit vectors $\mathbf{X}, \mathbf{Y}$ and $\mathbf{Z}$ of the global coordinate system expressed in the sensor frame $\mathrm{S}$

$$
{ }^{G S} \mathbf{R}=\left[\begin{array}{lll}
{ }^{S} \mathbf{X} & { }^{S} \mathbf{Y} & { }^{S} \mathbf{Z}
\end{array}\right]^{\mathrm{T}} .
$$

Gyroscope: The gyroscopes signals are described as the sum of the angular velocity $\boldsymbol{\omega}_{t}$, the offset $\mathbf{b}_{t}$, and a white noise term $\mathbf{v}_{G, t}$

$$
\mathbf{y}_{G, t}=\omega_{t}+\mathbf{b}_{t}+\mathbf{v}_{G, t} .
$$




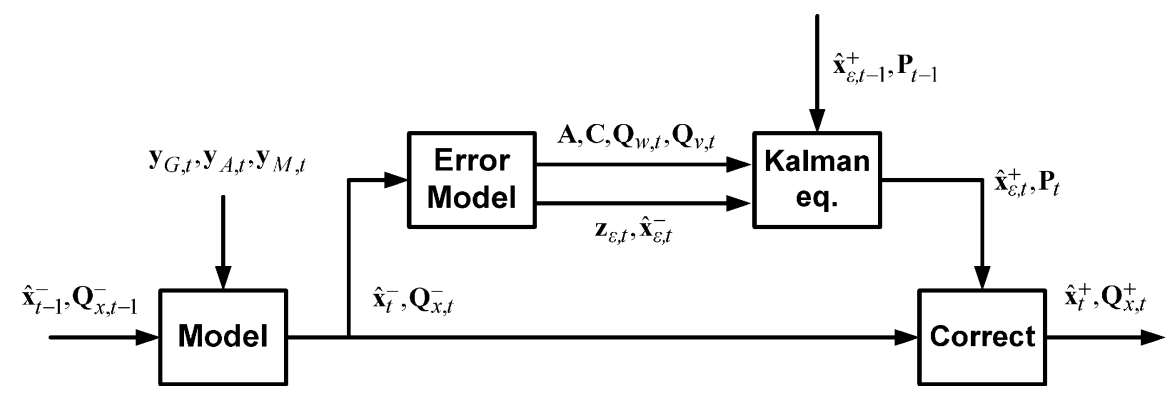

Fig. 2. Structure of complementary Kalman filter for orientation estimation. $\mathbf{x}$ is the vector featuring the states of the model used for the orientation estimation, whereas $\mathbf{x}_{\varepsilon}$ represents the errors in the model states: the gyroscope bias error, orientation error, and magnetic disturbance error. $\mathbf{Q}_{x}$ describes the covariance matrices of the filter states. Sensors signals are $\mathbf{y}_{G}$ : gyroscope; $\mathbf{y}_{A}$ : accelerometer; and $\mathbf{y}_{M}$ : magnetometer, from which the filter measurement input $\mathbf{z}_{\varepsilon}$ can be calculated using the error model. In the model, the angular velocities are integrated to an angular orientation and the $a$ priori signal predictions are made. A, $\mathbf{C}$, $\mathbf{Q}_{w}$, and $\mathbf{Q}_{v}$ are matrices describing the error model and $\mathbf{P}$ is the covariance matrix used in the Kalman filter.

The slow variation of the gyroscope offset is modeled as a realization of a first order Markov process, driven by a white Gaussian noise vector $\mathbf{w}_{b, t}$

$$
\mathbf{b}_{t}=\mathbf{b}_{t-1}+\mathbf{w}_{b, t} \text {. }
$$

Accelerometer: The accelerometer signals are described as the sum of the acceleration $\mathbf{a}_{t}$, the gravity $\mathbf{g}_{t}$, and a white noise term $\mathbf{v}_{A, t}$

$$
\mathbf{y}_{A, t}=\mathbf{a}_{t}-\mathbf{g}_{t}+\mathbf{v}_{A, t} .
$$

The acceleration was modeled as a first-order low-pass filtered white noise process according to

$$
\mathbf{a}_{t}=\mathrm{c}_{a} \cdot \mathbf{a}_{t-1}+\mathbf{w}_{a, t}
$$

where $0 \leq \mathrm{c}_{a}<1$, determining the cutoff frequency.

Magnetometer: The magnetometer signals are described as the sum of the earth magnetic field vector $\mathbf{m}_{t}$, a disturbance vector $\mathbf{d}_{t}$, and a white noise term $\mathbf{v}_{M, t}$ :

$$
\mathbf{y}_{M, t}=\mathbf{m}_{t}+\mathbf{d}_{t}+\mathbf{v}_{M, t} .
$$

The magnetic disturbance is modeled by the following Markov scheme

$$
\mathbf{d}_{t}=\mathrm{c}_{d} \cdot \mathbf{d}_{t-1}+\mathbf{w}_{d, t} .
$$

$\mathbf{w}_{d, t}$ is the driving Gaussian noise with a standard deviation of $\boldsymbol{\sigma}_{d}$ and $\mathrm{c}_{d}$ is a constant between 0 and 1 . In the vicinity of ferromagnetic materials or external magnetic fields, the magnetic flux is most likely to be higher or lower, as can be seen in Fig. 3 . In real 3-D space, the field distribution is of course more complicated, and, therefore, the magnetic dip angle $\varphi_{\text {mag }}$ should also be taken into account in order to identify a disturbance. The magnetic dip angle, also called magnetic inclination, is the angle the earth magnetic field makes with the surface of the earth.

This dip angle varies depending the position on the earth's surface [26]. The magnetic inclination $\varphi_{\mathrm{mag}}$ is $0^{\circ}$ at the magnetic equator and $90^{\circ}$ at each of the magnetic poles. In the latter case, magnetometers do not provide any heading information, which makes correction of gyroscope drift around the vertical

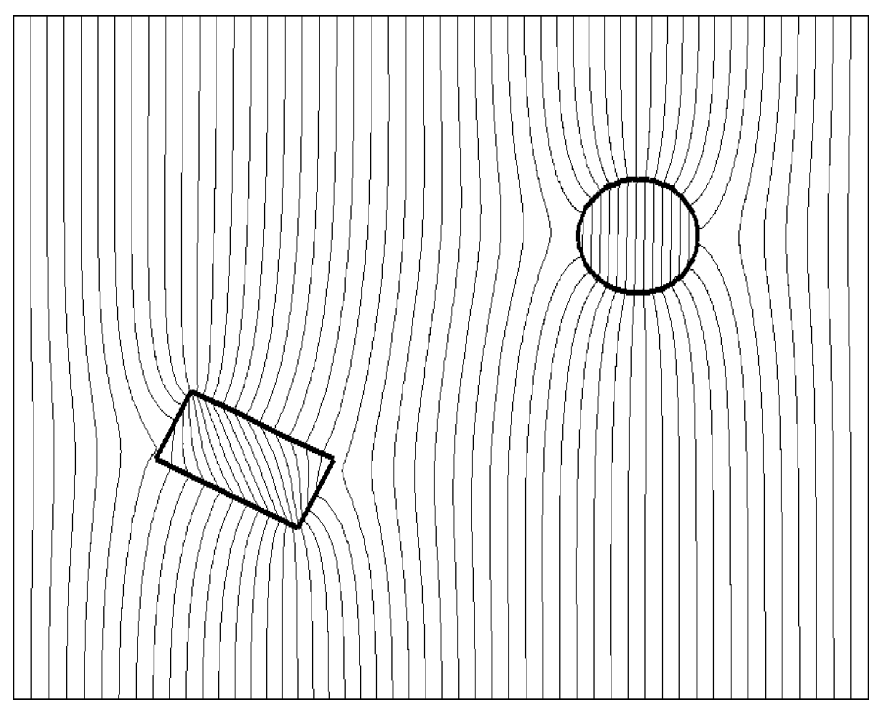

Fig. 3. Finite element simulation of ferromagnetic objects in free space with a homogeneus magnetic field. Objects have a permeability of 5000 times the permeability of free space $\left(4 \pi \times 10^{-7} \mathrm{H} / \mathrm{m}\right)$. Fieldlines bend toward the objects, generally favoring a perpendicular angle of incidence with the ferromagnetic surface.

axes impossible. At the locations of our experiments, the magnetic dip angle $\varphi_{\text {mag }}$ is about $67^{\circ}$. When changes in magnetic flux and dip angle are measured, $\boldsymbol{\sigma}_{d}$ will be increased, since this is the driving component in estimating the disturbance vector $\mathbf{d}_{t}$. The total flux $\left\|\mathbf{m}_{t}\right\|$ is calculated by taking the absolute value of the three magnetic sensor components

$$
\left\|\mathbf{m}_{t}\right\|=\sqrt{\mathrm{m}_{x, t}^{2}+\mathrm{m}_{y, t}^{2}+\mathrm{m}_{z, t}^{2}} .
$$

Under nondisturbed conditions, this value is normalized to $\left\|\mathbf{m}_{t}\right\|=1$. In order to calculate the magnetic dip angle, first, the measured magnetic sensor signals should be expressed in the global frame using

$$
{ }^{G} \mathbf{m}_{t}={ }^{G S} \mathbf{R}_{t} \cdot{ }^{S} \mathbf{m}_{t} .
$$

Then, the dip angle in the global frame is

$$
\varphi_{\mathrm{mag}, \mathrm{t}}=\arctan \left(\frac{{ }^{G} \mathrm{~m}_{z, t}}{\sqrt{{ }^{G} \mathrm{~m}_{x, t}^{2}+{ }^{G} \mathrm{~m}_{y, t}^{2}}}\right) .
$$


If $\left\|\mathbf{m}_{t}\right\|=1$ and $\varphi_{\text {mag,t }}=67^{\circ}$ there is a homogeneous magnetic field without ferromagnetic materials and the disturbance $\mathbf{d}_{t}$ equals zero

$$
\mathbf{d}_{t}=\left[\begin{array}{lll}
0 & 0 & 0
\end{array}\right]
$$

When $\left\|\mathbf{m}_{t}\right\|$ and $\varphi_{\text {mag,t }}$ change, $\mathbf{d}_{t}$ should change by updating $\sigma_{d}$

$$
\boldsymbol{\sigma}_{d}=\boldsymbol{\sigma}_{m}||\left|\mathbf{m}_{t}\|-\| \mathbf{m}_{t-1}\right| \|+\boldsymbol{\sigma}_{\varphi}\left|\varphi_{\text {mag,t }}-\varphi_{\text {mag,t-1 }}\right|
$$

where $\boldsymbol{\sigma}_{m}$ and $\boldsymbol{\sigma}_{\varphi}$ are vectors consisting of three equal components, which determine the contributions of the changes in total flux and dip angle, respectively.

\section{Filter Structure}

The Kalman filter uses a state space representation to model the relation between errors in estimated model variables and the error in the inclination and magnetic field vector predicted by the model. This relation is called an error state model and is governed by the linear stochastic difference equation [24]

$$
\mathbf{x}_{\varepsilon, t}=\mathbf{A} \mathbf{x}_{\varepsilon, t-1}+\mathbf{w}_{t}
$$

with a measurement $\mathbf{z}$ that is

$$
\mathbf{z}_{\varepsilon, t}=\mathbf{C x}_{\varepsilon, t}+\mathbf{v}_{t}
$$

$\mathbf{w}_{t}$ and $\mathbf{v}_{t}$ represent the system and measurement noise respectively. They are assumed to be independent, white and with normal probability distributions and are specified by the covariance matrices $\mathbf{Q}_{w, t}$ and $\mathbf{Q}_{v, t}$. Now $\mathbf{x}_{\varepsilon}, \mathbf{A}, \mathbf{z}_{\varepsilon}, \mathbf{C}$ and the covariance matrices have to be determined. The most important factors causing an error in the orientation estimate are incorporated in the error state vector $\mathbf{x}_{\varepsilon}$. The first error state variable is the orientation error, since it is used as a starting point to obtain the next orientation by strapdown integration. The second error state variable is the gyroscope offset error, since a small offset error causes a dramatic effect on the estimated orientation. The last error state variable is the error in the magnetic disturbance vector

$$
\mathbf{x}_{\varepsilon, t}=\left[\begin{array}{lll}
\boldsymbol{\theta}_{\varepsilon, t} & \mathbf{b}_{\varepsilon, t} & \mathbf{d}_{\varepsilon, t}
\end{array}\right]^{\mathrm{T}}
$$

The orientation error $\boldsymbol{\theta}_{\varepsilon}$ is defined as the angle and direction over which the actual sensor coordinate frame has to be rotated in order to coincide with the estimated sensor coordinate frame. Matrix $\mathbf{A}$ and noise component $\mathbf{w}_{t}$ describe the propagation of the a priori error state vector. They can be found by considering the effect of the unknown system components on the error state. In this complementary filter structure, the knowledge about previous errors is incorporated in the current state estimate. Consequently, there is no correlation between the $a$ priori estimated errors between two timesteps. This means that a priori errors $\mathbf{b}_{\varepsilon, t}^{-}, \boldsymbol{\theta}_{\varepsilon, t}^{-}$, and $\mathbf{d}_{\varepsilon, t}^{-}$do not depend on previous error states $\mathbf{b}_{\varepsilon, t-1}^{-}, \boldsymbol{\theta}_{\varepsilon, t-1}^{-}$and $\mathbf{d}_{\varepsilon, t-1}^{-}$. Therefore, the $\mathbf{A}$ matrix equals the zero matrix.
The error estimate is updated with measurement information according to

$$
\hat{\mathbf{x}}_{\varepsilon, t}^{+}=\hat{\mathbf{x}}_{\varepsilon, t}^{-}+\mathbf{K}_{t}\left(\mathbf{z}_{\varepsilon, t}-\mathbf{C} \hat{\mathbf{x}}_{\varepsilon, t}^{-}\right)
$$

where the Kalman gain matrix $\mathbf{K}$ is computed from the estimation error covariance matrix, according to

$$
\mathbf{K}_{t}=\mathbf{P}_{t}^{-} \mathbf{C}^{\mathrm{T}}\left(\mathbf{C P}_{t}^{-} \mathbf{C}^{\mathrm{T}}+\mathbf{Q}_{v, t}\right)^{-1}
$$

and $\mathbf{P}$ is updated according to the Ricatti equation

$$
\mathbf{P}_{t+1}=\mathbf{A}\left(\mathbf{I}-\mathbf{K}_{t} \mathbf{C}\right) \mathbf{P}_{t} \mathbf{A}^{\mathrm{T}}+\mathbf{Q}_{w, t+1} .
$$

Since matrix A equals zero, the Ricatti equation simplifies to

$$
\mathbf{P}_{t+1}=\mathbf{Q}_{w, t+1} \text {. }
$$

Matrix $\mathbf{C}$ and noise $\mathbf{v}_{t}$ describe the relation between the error states and the measurements (Kalman filter input). They can be found by considering the effect of the gyroscope offset, orientation error, and magnetic disturbance on the inclination and magnetic vector estimates. The filter has therefore two different inputs. A sensor signal generation model was developed to make two estimates of inclination [23], one based on the gyroscope signals $\left(\mathbf{Z}_{G}\right)$ and one based on the accelerometer signals $\left(\mathbf{Z}_{A}\right)$ (see Fig. 1)

$$
\mathbf{z}_{\varepsilon, \text { inclination, } \mathrm{t}}={ }^{S} \hat{\mathbf{Z}}_{A, t}-{ }^{S} \hat{\mathbf{Z}}_{G, t} .
$$

The same principle of the inclination sensor signals generation model was applied for the estimation of the global magnetic vector. Both magnetometer $\left(\mathbf{H}_{M}\right)$ and gyroscope $\left(\mathbf{H}_{G}\right)$ systems make an estimate of the magnetic field vector

$$
\mathbf{z}_{\varepsilon, \text { magnetic }, \mathrm{t}}={ }^{S} \hat{\mathbf{H}}_{M, t}-{ }^{S} \hat{\mathbf{H}}_{G, t} \text {. }
$$

The error input can be formed by combining (20) and (21) in one vector

$$
\mathbf{z}_{\varepsilon, t}=\left[\begin{array}{ll}
\mathbf{z}_{\varepsilon, \text { incl }, \mathrm{t}} \quad \mathbf{z}_{\varepsilon, \mathrm{mag}, \mathrm{t}}
\end{array}\right]^{\mathrm{T}}
$$

The inclination estimate from the accelerometer is calculated by subtracting the predicted acceleration $\hat{a}_{t}^{-}$from the accelerometer signal to obtain the gravity vector. The gravity vector is normalized to obtain an estimate of the inclination of $S \hat{\mathbf{Z}}_{A, t}^{-}$

$$
\begin{aligned}
{ }^{S} \hat{\mathbf{Z}}_{A, t}^{-} & =\frac{\mathbf{y}_{A, t}-{ }^{S} \hat{\mathbf{a}}_{t}^{-}}{\left|\mathbf{y}_{A, t}-S \hat{\mathbf{a}}_{t}^{-}\right|} \\
& ={ }^{S} \mathbf{Z}_{t}+\frac{1}{g}\left(-{ }^{S} \hat{\mathbf{a}}_{t} \times \hat{\boldsymbol{\theta}}_{\varepsilon, t}^{-}-\mathrm{c}_{a} \cdot{ }^{S} \hat{\mathbf{a}}_{\varepsilon, t-1}^{+}+\mathbf{w}_{a, t}+\mathbf{v}_{A, t}\right)
\end{aligned}
$$

with ${ }^{S} \mathbf{Z}_{t}$ being the correct inclination vector at time t, ${ }^{S} \hat{\mathbf{a}}_{t} \times \hat{\boldsymbol{\theta}}_{\varepsilon, t}^{-}$ the effect of the orientation error on the acceleration estimate and $\mathrm{c}_{a} \cdot{ }^{S} \hat{\mathbf{a}}_{\varepsilon, t-1}^{+}$the a priori acceleration error. The estimate of ${ }^{S} \hat{\mathbf{H}}_{M, t}^{-}$is the measured magnetic vector subtracted by the estimated magnetic disturbance vector ${ }^{S} \hat{\mathbf{d}}_{t}^{-}$

$$
\begin{aligned}
{ }^{S} \hat{\mathbf{H}}_{M, t}^{-} & =\mathbf{y}_{M, t}-{ }^{S} \hat{\mathbf{d}}_{t}^{-} \\
& ={ }^{S} \mathbf{H}_{t}-{ }^{S} \hat{\mathbf{d}}_{t} \times \hat{\boldsymbol{\theta}}_{\varepsilon, t}^{-}-\mathrm{c}_{d} \cdot{ }^{S} \hat{\mathbf{d}}_{\varepsilon, t-1}^{+}+\mathbf{w}_{d, t}+\mathbf{v}_{M, t}
\end{aligned}
$$

with ${ }^{S} \mathbf{H}_{t}$ being the correct normalized magnetic vector at time $\mathrm{t},{ }^{S} \hat{\mathbf{d}}_{t} \times \hat{\boldsymbol{\theta}}_{\varepsilon, t}^{-}$the effect of the orientation error on the mag- 
netic disturbance estimate, and $\mathrm{c}_{d} \cdot S \hat{\mathbf{d}}_{\varepsilon, t-1}^{+}$the a priori magnetic disturbance error. To define the inclination estimate ${ }^{S} \hat{\mathbf{Z}}_{G, t}^{-}$ and magnetic vector ${ }^{S} \hat{\mathbf{H}}_{G, t}^{-}$from the gyroscopes, first the orientation after one integration step has to be calculated. Because the error in predicted error states are small compared to the actual signals, only first order approximation of the error model is made. For small errors, the relation between the actual and estimated orientation is given by [25]

$$
{ }^{G S} \hat{\mathbf{R}}={ }^{G S} \mathbf{R} \cdot\left(\mathbf{I}+\left[\boldsymbol{\theta}_{\varepsilon} \times\right]\right) .
$$

The matrix cross product operator is given by

$$
[\boldsymbol{\theta} \times]=\left[\begin{array}{ccc}
0 & -\theta_{z} & \theta_{y} \\
\theta_{z} & 0 & -\theta_{x} \\
-\theta_{y} & \theta_{x} & 0
\end{array}\right]
$$

The orientation can be found by considering a first order approximation of a strapdown integration step

$$
{ }^{G S} \hat{\mathbf{R}}_{t}^{-}={ }^{G S} \mathbf{R}_{t-1}^{+}+{ }^{G S} \mathbf{R}_{t-1}^{+} \cdot\left[T \cdot \hat{\boldsymbol{\omega}}_{t}^{-} \times\right]
$$

where $T$ is the sample time. By substituting the angular velocity estimate from (2) into the previous equation and neglecting products of errors, it follows that the error propagation $\boldsymbol{\theta}_{\varepsilon, t}$ is described by

$$
\boldsymbol{\theta}_{\varepsilon, t}^{-}=\boldsymbol{\theta}_{\varepsilon, t-1}^{+}-T \cdot \mathbf{b}_{\varepsilon, t-1}^{+}+T \cdot \mathbf{v}_{G, t} \cdot
$$

For the inclination estimate, the strapdown integration can be approximated with

$$
\begin{aligned}
S \hat{\mathbf{Z}}_{G, t}^{-}= & { }^{S} \hat{\mathbf{Z}}_{t-1}^{+}-T \cdot{ }^{S} \hat{\boldsymbol{\omega}}_{t} \times{ }^{S} \hat{\mathbf{Z}}_{t-1}^{+} \\
\approx & { }^{S} \mathbf{Z}_{t}+{ }^{S} \hat{\mathbf{Z}}_{t-1} \times \hat{\boldsymbol{\theta}}_{\varepsilon, t-1}^{+}-T \cdot{ }^{S} \hat{\mathbf{Z}}_{t-1} \\
& \times \hat{\mathbf{b}}_{\varepsilon, t-1}^{+}+{ }^{S} \hat{\mathbf{Z}}_{t-1} \times T \cdot \mathbf{v}_{G, t} .
\end{aligned}
$$

The gyroscope-based estimated magnetic vector is obtained similarly to the gyroscope-based inclination

$$
\begin{aligned}
{ }^{S} \hat{\mathbf{H}}_{G, t}^{-}= & { }^{S} \hat{\mathbf{H}}_{t-1}^{+}-T \cdot{ }^{S} \hat{\boldsymbol{\omega}}_{t} \times{ }^{S} \hat{\mathbf{H}}_{t-1}^{+} \\
\approx & { }^{S} \mathbf{H}_{t}+{ }^{S} \hat{\mathbf{H}}_{t-1} \times \hat{\boldsymbol{\theta}}_{\varepsilon, t-1}^{+}-T \cdot{ }^{S} \hat{\mathbf{H}}_{t-1} \\
& \times \hat{\mathbf{b}}_{\varepsilon, t-1}^{+}+{ }^{S} \hat{\mathbf{H}}_{t-1} \times T \cdot \mathbf{v}_{G, t} .
\end{aligned}
$$

Now, the $\mathbf{C}$ matrix can be defined

$$
\begin{aligned}
\mathbf{z}_{\varepsilon, t} & =\left[\begin{array}{c}
{ }^{S} \mathbf{Z}_{A, t}-{ }^{S} \mathbf{Z}_{G, t} \\
{ }^{S} \mathbf{H}_{M, t}-{ }^{S} \mathbf{H}_{G, t}
\end{array}\right] \\
& =\mathbf{C} \cdot\left\{\begin{array}{l}
\boldsymbol{\theta}_{\varepsilon, t} \\
\mathbf{b}_{\varepsilon, t} \\
\mathbf{d}_{\varepsilon, t}
\end{array}\right\}+\mathbf{v}_{t}
\end{aligned}
$$

with $\mathbf{C}$ a $6 * 9$ matrix

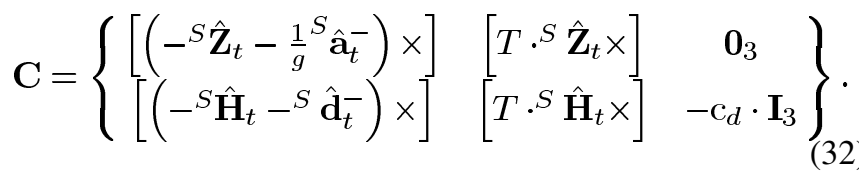

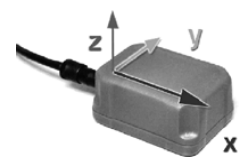

Fig. 4. MT9-A module consisting of 3-D gyroscopes, 3-D accelerometers, 3-D magnetometers, and a temperature sensor. Dimensions of the module are $39 *$ $54 * 28 \mathrm{~mm}(\mathrm{~W} * \mathrm{~L} * \mathrm{H})$ and the weight is $40 \mathrm{~g}$. Sensor frame is indicated by XYZ.

$\mathbf{I}_{3}$ is the $3 \times 3$ identity matrix and $\mathbf{0}_{3}$ the $3 \times 3$ zero matrix. The noise term $\mathbf{v}_{t}$ is described by

$\mathbf{v}_{t}=\left\{\begin{array}{c}\frac{1}{g}\left(-\mathrm{c}_{a} \cdot{ }^{S} \hat{\mathbf{a}}_{\varepsilon, t-1}^{+}+\mathbf{v}_{A, t}+\mathbf{w}_{a, t}\right)-{ }^{S} \hat{\mathbf{Z}}_{t} \times T \cdot \mathbf{v}_{G, t} \\ \mathbf{w}_{d, t}+\mathbf{v}_{M, t}-{ }^{S} \hat{\mathbf{H}}_{t} \times T \cdot \mathbf{v}_{G, t}\end{array}\right\}$.

\section{Covariance Matrices}

The error covariance matrix $\left(\mathbf{Q}_{w, t}\right)$ of the system noise term $\mathbf{w}_{t}$ can be found using the knowledge that the $\mathbf{A}$ matrix equals the zero matrix and by taking the variances of the error propagations (28), as shown in (34) at the bottom of the page. where $\mathbf{Q}_{\theta, t-1}^{+}, \mathbf{Q}_{b, t-1}^{+}$and $\mathbf{Q}_{d, t-1}^{+}$are the a posteriori error covariance matrices of the orientation, offset and magnetic disturbance at the previous timestep. $\mathbf{Q}_{v G, t}$ is the gyroscope noise covariance matrix, $\mathbf{Q}_{w b, t}$ the covariance matrix of the offset noise $\mathbf{w}_{b, t}$ and $\mathbf{Q}_{w d, t}$ the covariance matrix of the driving noise $\mathbf{w}_{d, t}$ of the magnetic disturbance. The term $\mathbf{Q}_{v G, t}$ was found by assuming that the gyroscope noise variance is equal in the $\mathrm{x}-, \mathrm{y}-$, and $\mathrm{z}$-direction. In this case, the noise covariance matrix does not change when the noise is expressed in a different reference system. The measurement noise covariance $\mathbf{Q}_{v, t}$ was found by taking the covariances of (33)

$$
\mathbf{Q}_{v, t}=\left\{\begin{array}{c}
\frac{1}{g^{2}}\left(\mathrm{c}_{a}^{2} \cdot \mathbf{Q}_{a, t-1}^{+}+\mathbf{Q}_{w a, t}+\mathbf{Q}_{v A, t}\right)+\mathbf{Q}_{v G, t} \\
\mathbf{Q}_{w d, t}+\mathbf{Q}_{v M, t}+\mathbf{Q}_{v G, t}
\end{array}\right\}
$$

with $\mathbf{Q}_{a, t-1}^{+}$the a posteriori acceleration error covariance matrix, $\mathbf{Q}_{w a, t}$ the covariance matrix of $\mathbf{w}_{a, t}, \mathbf{Q}_{v A, t}$ the covariance of the accelerometer measurement noise vector $\mathbf{v}_{A, t}$ and $\mathbf{Q}_{v M, t}$ the covariance of the magnetometer measurement noise vector $\mathbf{v}_{M, t}$.

\section{E. Experimental Methods}

The purpose of the experiments was to investigate the accuracy, stability, and reproducibility of the orientation estimation under various conditions. For the experiments, a MT9-A (Xsens Motion Technologies [27], see Fig. 4) inertial and magnetic sensor module was used. The module consisted of three orthogonally placed angular rate sensors (Murata ENC03J), 3-D linear acceleration sensors (Analog Devices ADXL202E), 3-D magnetoresistive sensors (Philips KMZ51 and KMZ52), and an ambient temperature sensor. All sensor signals were sampled at

$$
\mathbf{Q}_{w, t}=\left[\begin{array}{ccc}
\mathbf{Q}_{\theta, t-1}^{+}+T^{2} \cdot \mathbf{Q}_{b, t-1}^{+}+T^{2} \cdot \mathbf{Q}_{v G, t} & T^{2} \cdot \mathbf{Q}_{b, t-1}^{+} & 0 \\
T^{2} \cdot \mathbf{Q}_{b, t-1}^{+} & \mathbf{Q}_{b, t-1+}^{+} \mathbf{Q}_{w b, t} & 0 \\
0 & 0 & c_{d}^{2} \mathbf{Q}_{d, t-1}^{+}+\mathbf{Q}_{w d, t}
\end{array}\right]
$$



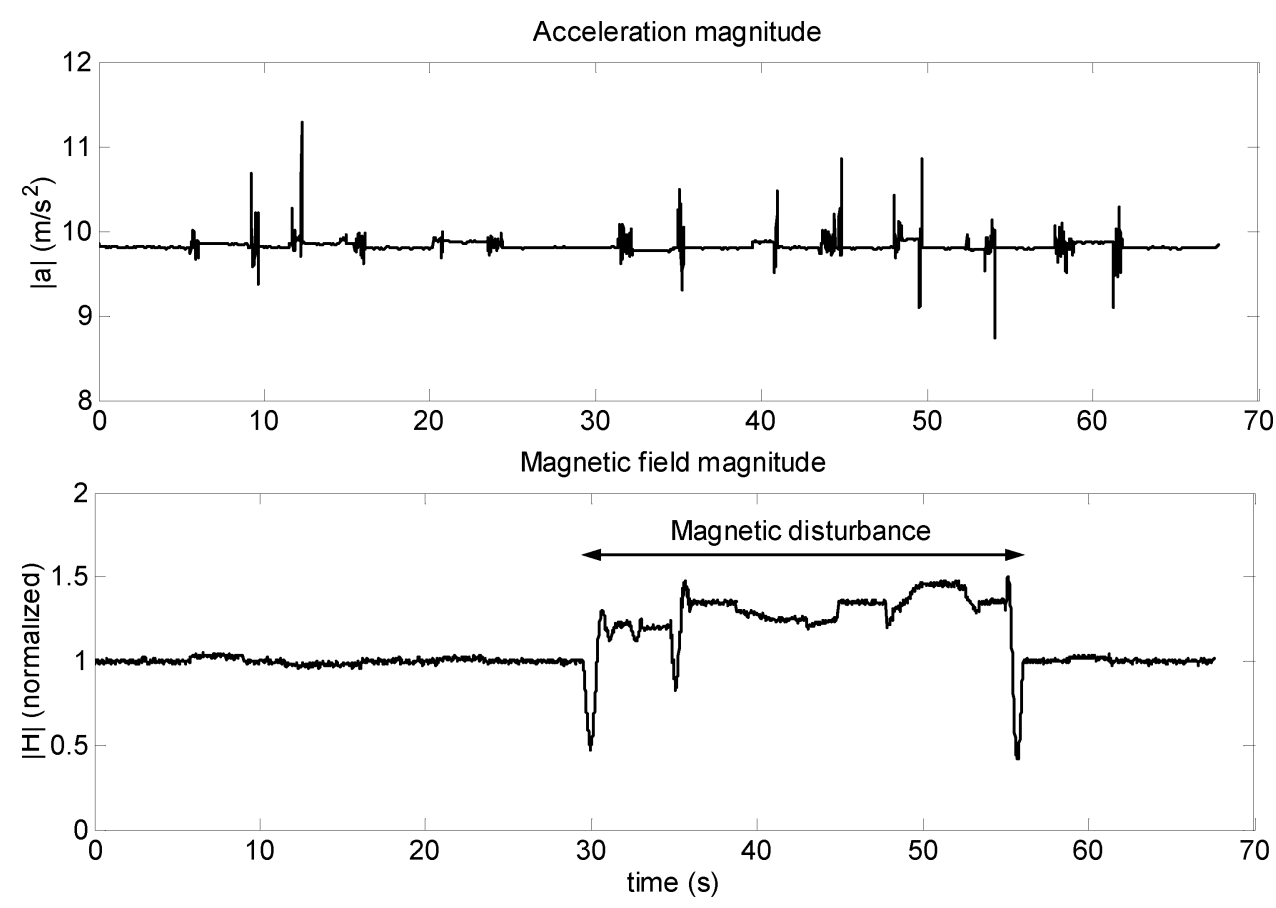

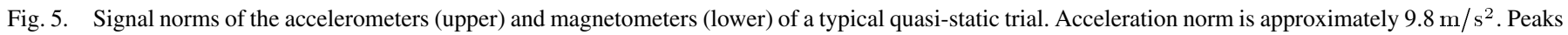

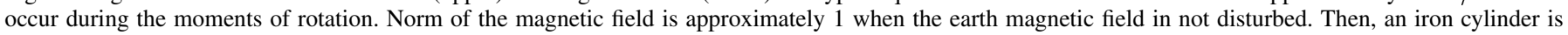
placed near the sensor module from 30 to $55 \mathrm{~s}$ and the disturbance can be detected. After the cylinder is removed, the norm is 1 again.

$100 \mathrm{~Hz}$ with 16 bits. An anti-aliasing filter of $50 \mathrm{~Hz}$ was applied to the gyroscopes and accelerometers and magnetometers were low-passed filtered at $10 \mathrm{~Hz}$, all with second order filters. The calibration procedure to obtain the gains, offsets and nonorthogonality of accelerometers, gyroscopes and magnetometers was performed by the manufacturer of the sensor module and was based on Ferraris [28].

The stability of the filter was first investigated under static conditions. An iron cylinder of $3.75 \mathrm{~kg}$ was placed near the sensor module for $10 \mathrm{~min}$ without moving the sensor. The second quasi-static tests implied rotations of + and $-90^{\circ}$ along the three axes. To ensure exact angles of rotations, the sensor module was fixed in an aluminum (not ferromagnetic) cubic frame. The definition of the sensor frame $S$ can be found in Fig. 4. After each rotation, the module was not moved for $2-4$ s. The sensor module was first rotated $90^{\circ}$ and $-90^{\circ}$ along the $\mathrm{x}$-axis, followed by $-90^{\circ}$ and $90^{\circ}$ along the $\mathrm{y}$-axis. It was then rotated $90^{\circ}$ along the z-axis, and $-90^{\circ}$ back to its original orientation. After these rotations, the iron cylinder was placed at $5 \mathrm{~cm}$ of the module and a new sequence of rotations was performed in opposite directions. The iron was then taken away and the sensor was rotated $90^{\circ}$ along the $\mathrm{x}$ axis and $-90^{\circ}$ back. The angles as calculated by the Kalman filter were compared with the physical orientation of the sensor in the aluminum frame. All these experiments were repeated ten times. In the third experiment, the orientation as calculated by the filter was compared with the orientation that was obtained by a laboratory bound 3-D optical tracking system Vicon 370 (six cameras, $50 \mathrm{~Hz}$, Oxford Metrics). Three optical markers were attached orthogonally to the sensor module on $10-\mathrm{cm}$ carbon fiber sticks in order to measure the orientation of the sensor. The orientation obtained with the Vicon system was synchronized and resampled to coincide with the orientation estimated by the Kalman filter. The sensor module with the attached markers was placed on a 50-cm-long stick and moved by hand near a large iron tool case. The movements consisted of small and large rotations along multiple axes at different velocities and different distances from the ferromagnetic case.

Before using the filter, the model parameter $c_{a}$ was estimated by analyzing typical accelerations [23] of several movements. The parameter $c_{d}$ was obtained by characterizing the disturbances by moving the sensor module at different speeds and distances from ferromagnetic materials. The sensor noise variances $\mathbf{Q}_{v A}, \mathbf{Q}_{v G}$, and $\mathbf{Q}_{v M}$ were found by taking the variances of the sensor signals while the sensor was lying still. These parameters were not changed during the experiments.

\section{REsUlts}

The 10-min static tests showed no drift or interference problems. The accuracy was $0.6^{\circ}$ root means square (rms) (standard deviation 0.3 ). Fig. 5 shows the signal norms of the accelerometers and magnetometers of one typical trial of the quasi-static experiments. The acceleration norm shows a constant value of approximately $9.8 \mathrm{~m} / \mathrm{s}^{2}$ with peaks at the moments of rotation. The magnetic norm has a value of approximately 1 when no iron is near the sensor. When the iron mass is moved toward the sensor (marked by the arrow), the magnetic disturbance can be detected.

In Fig. 6, the Euler angles along the three axes are given when only the angular velocities from the gyroscopes are integrated. It can be seen that the integration drift is between $10^{\circ}-25^{\circ}$ after $1 \mathrm{~min}$. Although the calculations for orientations are not performed using Euler angles, for obvious reasons like 


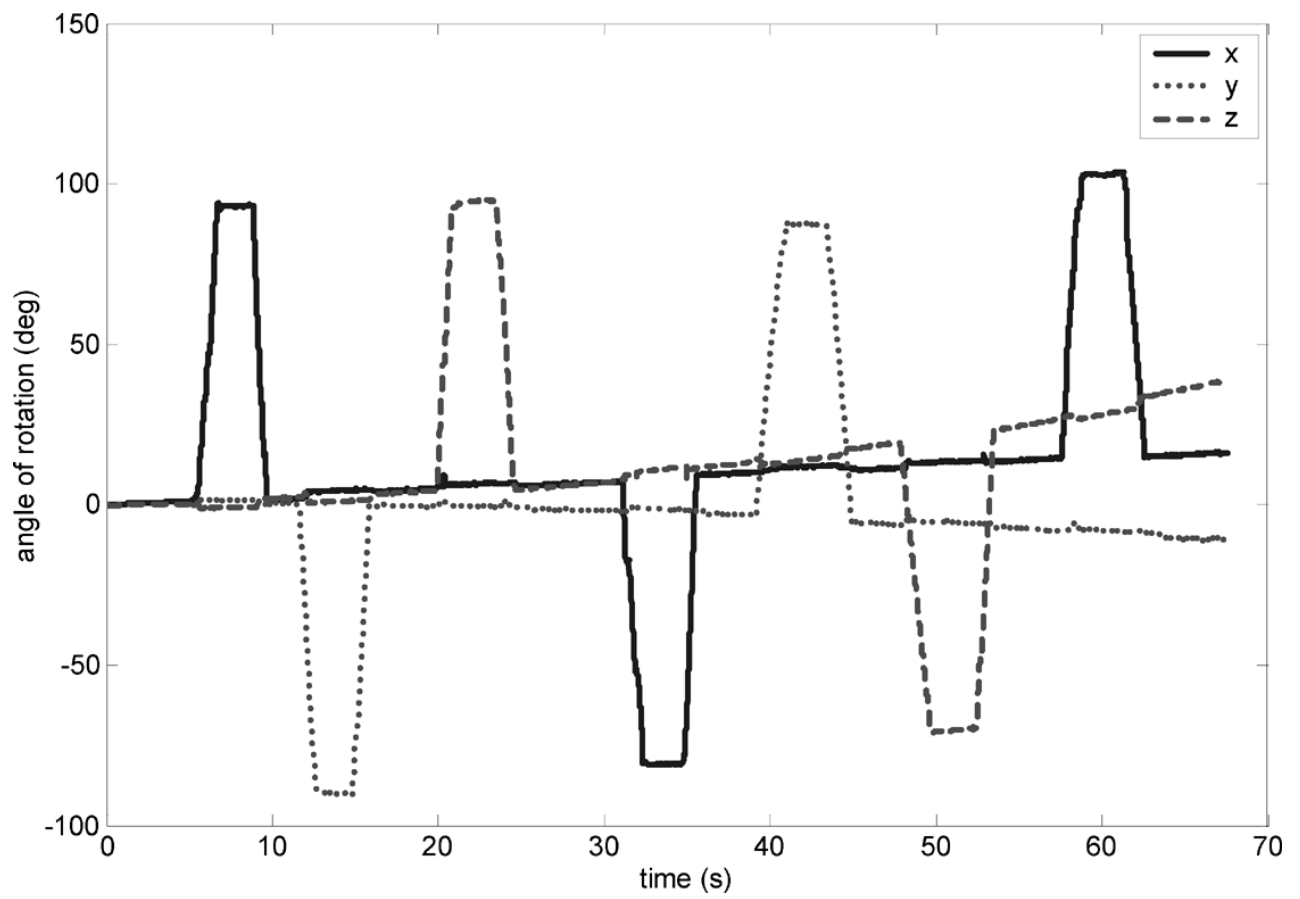

Fig. 6. Euler angle presentation of rotations around the $\mathrm{x}$ (solid), $\mathrm{y}$ (dot), and $\mathrm{z}$ (dashed) axes when only the gyroscope angular velocities are integrated. After a few seconds, the drift error becomes significant.

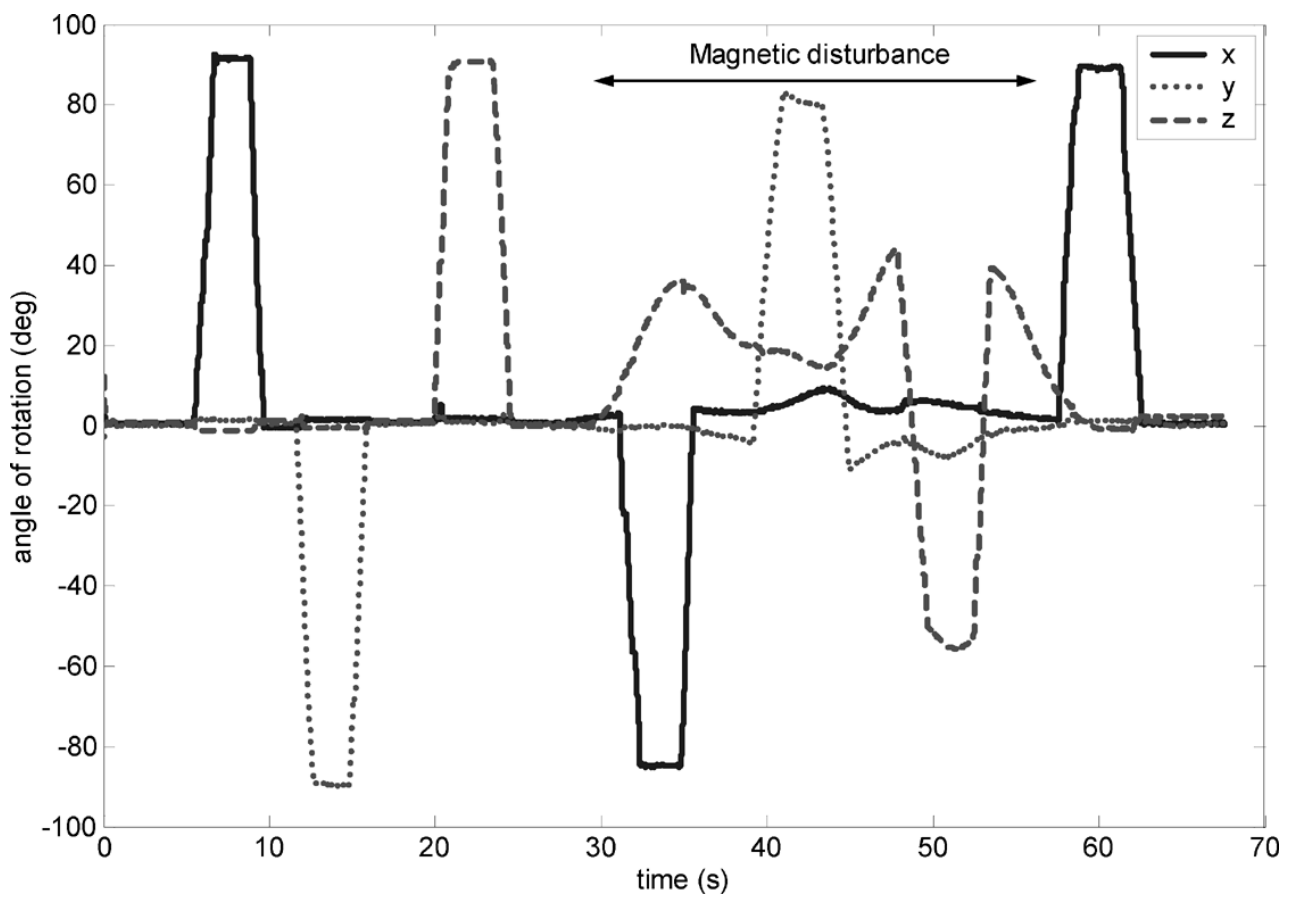

Fig. 7. Angles of rotation with a Kalman filter with equal weight to gyroscopes, accelerometers, and magnetometers. No magnetic disturbance compensation is applied and the errors become quite large during the period of interference (marked by the arrow).

singularities, these results are presented in this way for better interpretation.

Obviously, there is no magnetic disturbance noticeable since the gyroscopes are not interfered by ferromagnetic materials. Fig. 7 shows the output from the same motion sequence when a Kalman filter is used with all three types of sensors but no magnetic disturbance compensation is applied. From the start of the interference, the error becomes significant.
When the iron is removed, the rotation around the z-axis slowly convergences back to its original orientation. The interference can also be observed in the $\mathrm{X}$ and $\mathrm{Y}$ (inclination) components, because of the influence on the magnetic dip angle.

Fig. 8 illustrates the effect of the magnetic signal model and the Kalman filter on the same signals. The output of the Kalman filter is drift-free, not disturbed by the iron and the rotations are estimated accurately. 


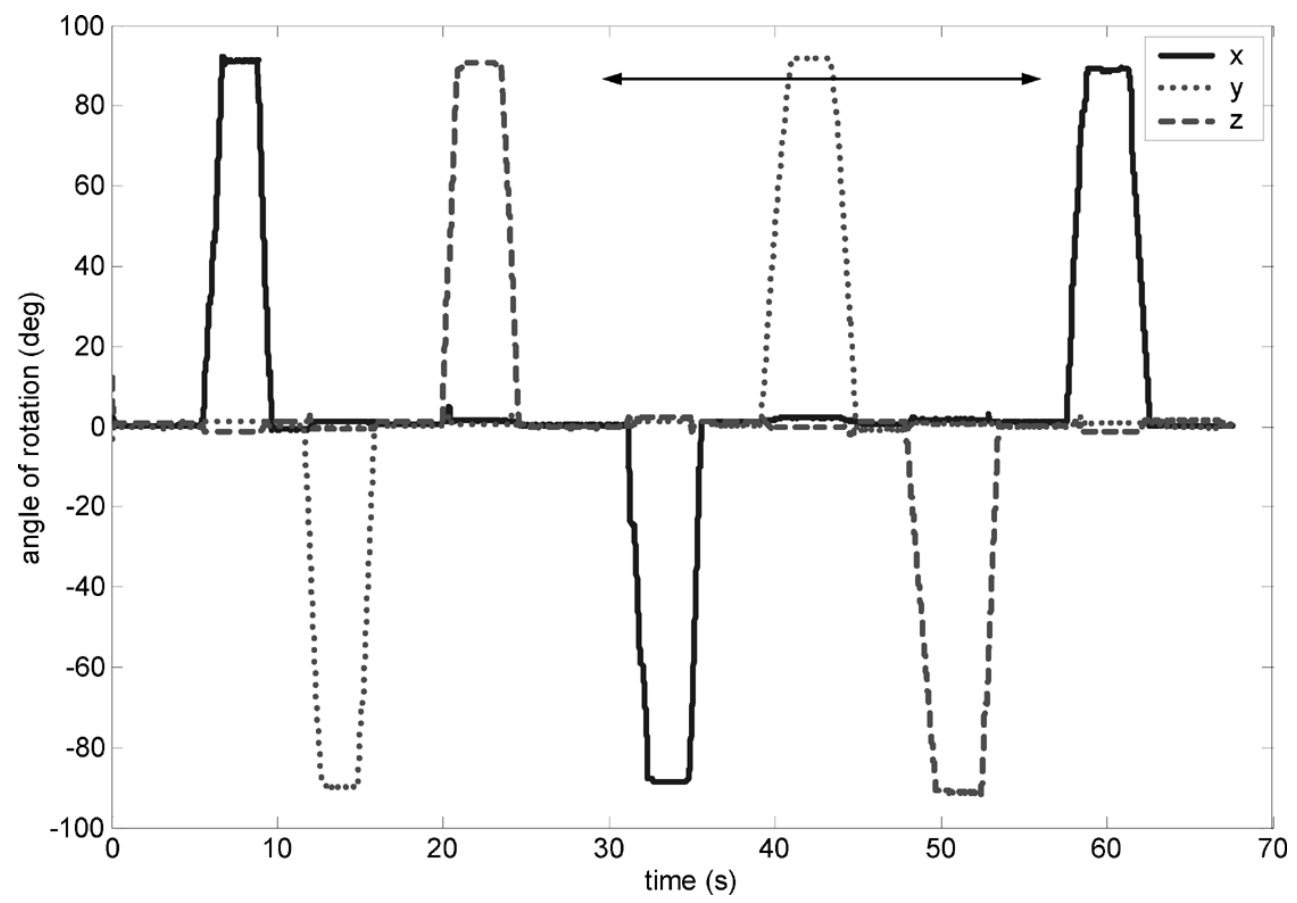

Fig. 8. Angles of rotation with the full Kalman filter featuring the magnetic disturbance compensation. During the period of interference (marked by the arrow), the output is not disturbed and the whole trial is drift-free.

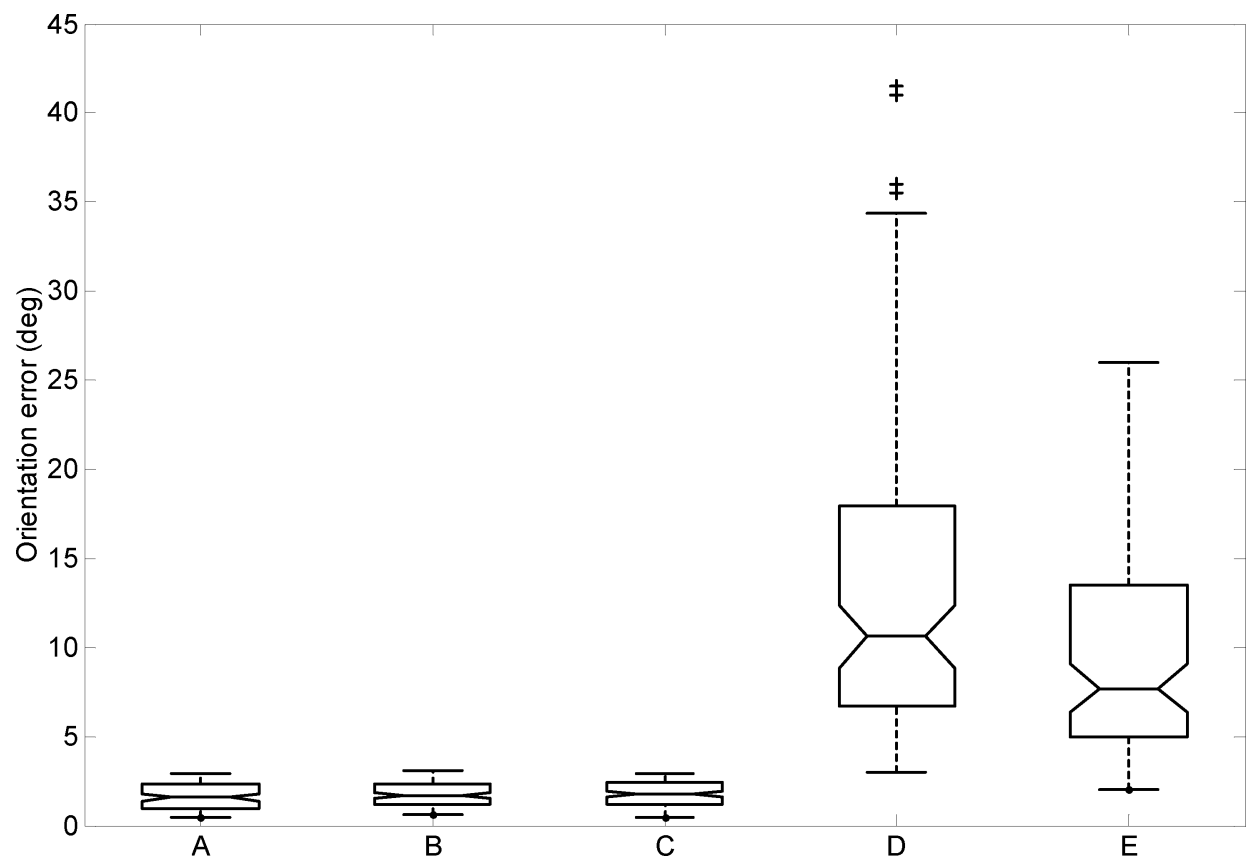

Fig. 9. Orientation estimation errors of quasi-static experiments with magnetic interference presented in box plots. Boxes have lines at the lower quartile, median, and upper quartile values. Whiskers are lines extending from each end of the box to show the extent of the rest of the data. Outliers are marked with the + signs (some are beyond boundaries of graph). Left box A shows the static errors of the full Kalman filter with magnetic disturbance compensation during the parts when no ferromagnetic materials were near the sensor. Box B shows the results of the full Kalman filter during the parts without magnetic disturbances. In the middle box C, the errors are shown where a Kalman filter was used without compensation and no disturbances were present. In box D, it can be seen that the Kalman filter without magnetic disturbance compensations has big errors. In the right box E, the errors are presented when only the gyroscope signals are integrated using a strapdown integration algorithm.

In Fig. 9, the distribution of the errors of the quasi-static experiments is presented in box plots. The errors are defined as the angle over which the filter output has to be rotated to coincide with the actual angles of rotation of the sensor in the frame during all static parts. The first box A shows the static errors of the full Kalman filter with magnetic disturbance compensation during the parts when no ferromagnetic materials were near the sensor. It was normally distributed with a mean of $1.3^{\circ}$ and a standard deviation of 0.4. Box B shows the results of the full Kalman filter during the parts where the magnetic field was disturbed by the iron object. The mean error was now $1.5^{\circ}$ (standard deviation 0.45). In the middle box $\mathrm{C}$, the errors are shown 

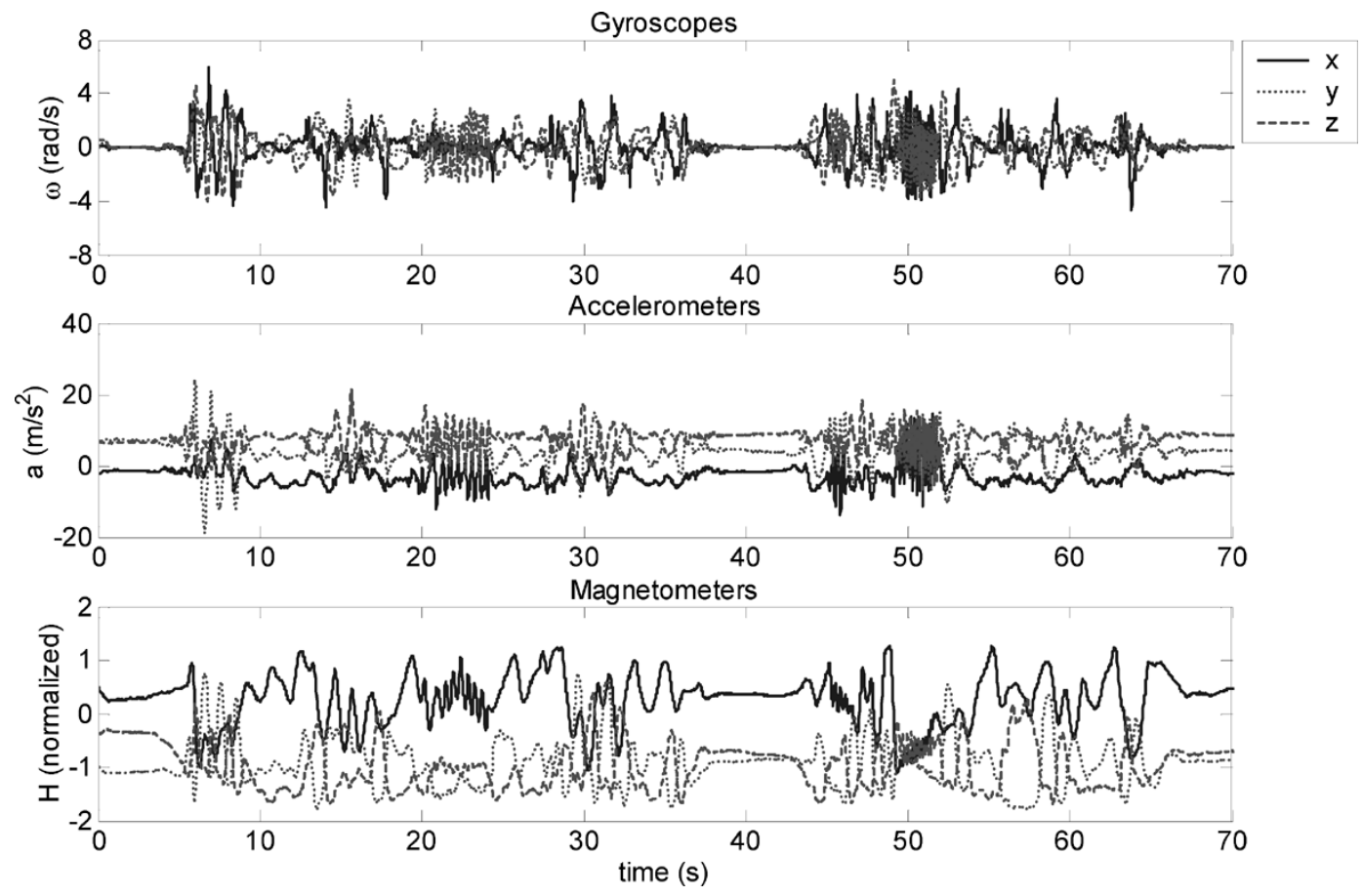

Fig. 10. Sensor signals of gyroscopes (upper), accelerometers (middle) and magnetometers (lower) of combined 3-D rotations near the ferromagnetic box.

where a Kalman filter was used without compensation and no disturbances were present. The errors were equal to the full Kalman filter without the disturbance, namely $1.3^{\circ}$ (standard deviation 0.4). In box D, it can be seen that the Kalman filter without magnetic disturbance compensation had big errors up to $40^{\circ}$ when iron is placed near the sensor module. In many practical applications, this will cause big problems. The errors, when only the angular velocities of the gyroscopes were integrated, are plotted in the fifth bar E.

It should be noted that the error in gyroscope integration is depending on the length of the trial. Increasing the duration of the trial will increase the gyroscope drift error. There was a significant difference (Friedman, Anova, and posthoc test Wilcoxon, $p<0.01$ ) between the orientation estimates with compensation and the orientation estimates without compensation and only gyroscope integration at the periods of magnetic interference. Between A, B, and C, no significant differences were found.

The gyroscope, accelerometer and magnetometer signals of the third experiment are presented in Fig. 10. The gyroscopes signals show the angular velocities of the 3 -D rotations. The accelerometers show the three components of the gravitational acceleration and the acceleration of the sensor. The components of the disturbed magnetic field vector as measured by the magnetometers are plotted in the lower graph.

In Fig. 11, the norms of the accelerometer and magnetometer signals are given in the upper and middle graph, respectively. The effect of the magnetic disturbance is clearly noticeable in variability of the magnetic norm. The difference in orientation estimated with the inertial and magnetic sensor module compared to the optical reference system is given in the lower graph. The error was expressed by the three components of the difference vector between both orientation estimates and was $2.7^{\circ}$ rms. When no magnetic disturbance compensation was applied the error was $11.9^{\circ} \mathrm{rms}$. The Vicon system was considered to have an accuracy of $1^{\circ} \mathrm{rms}$ [29], [30].

\section{DISCUSSION}

This paper proposes a method for fusion of gyroscope, accelerometer, and magnetometer signals to estimate orientation of human body segments. The combination of the three types of sensor signals for human motion analysis has been reported previously [12], [14], [20], [21]. However, magnetic interference has not been taken into account in these filters and large errors will occur in the vicinity of ferromagnetic objects. The performances of the method proposed in this paper have been tested under static, quasi-static, and dynamic conditions and evaluated with an external optical reference system for the dynamic experiments. The results show that the orientation estimates significantly improve using the magnetic interference correction and the filter overcomes both sensor and electronics drift. The structure of the complementary filter, where only three factors $\left(\boldsymbol{\theta}_{\varepsilon}, \mathbf{b}_{\varepsilon}\right.$, and $\left.\mathbf{d}_{\varepsilon}\right)$ have to be estimated enables fast real time implementation.

From the experiments and literature [31], it can be found that the accuracy of the dynamic orientation measurements is sensitive to several factors, namely, the speed and type of movement, often depending on the body segment on which the sensor module is placed and the environment, which means the distance to the ferromagnetic material, type, mass, and geometry. In this study, the orientation filter was tested under controlled and limited conditions. To further assess the system, 3-D movements of different body segments should be compared with an external tracking reference system in different magnetically disturbed surroundings. 

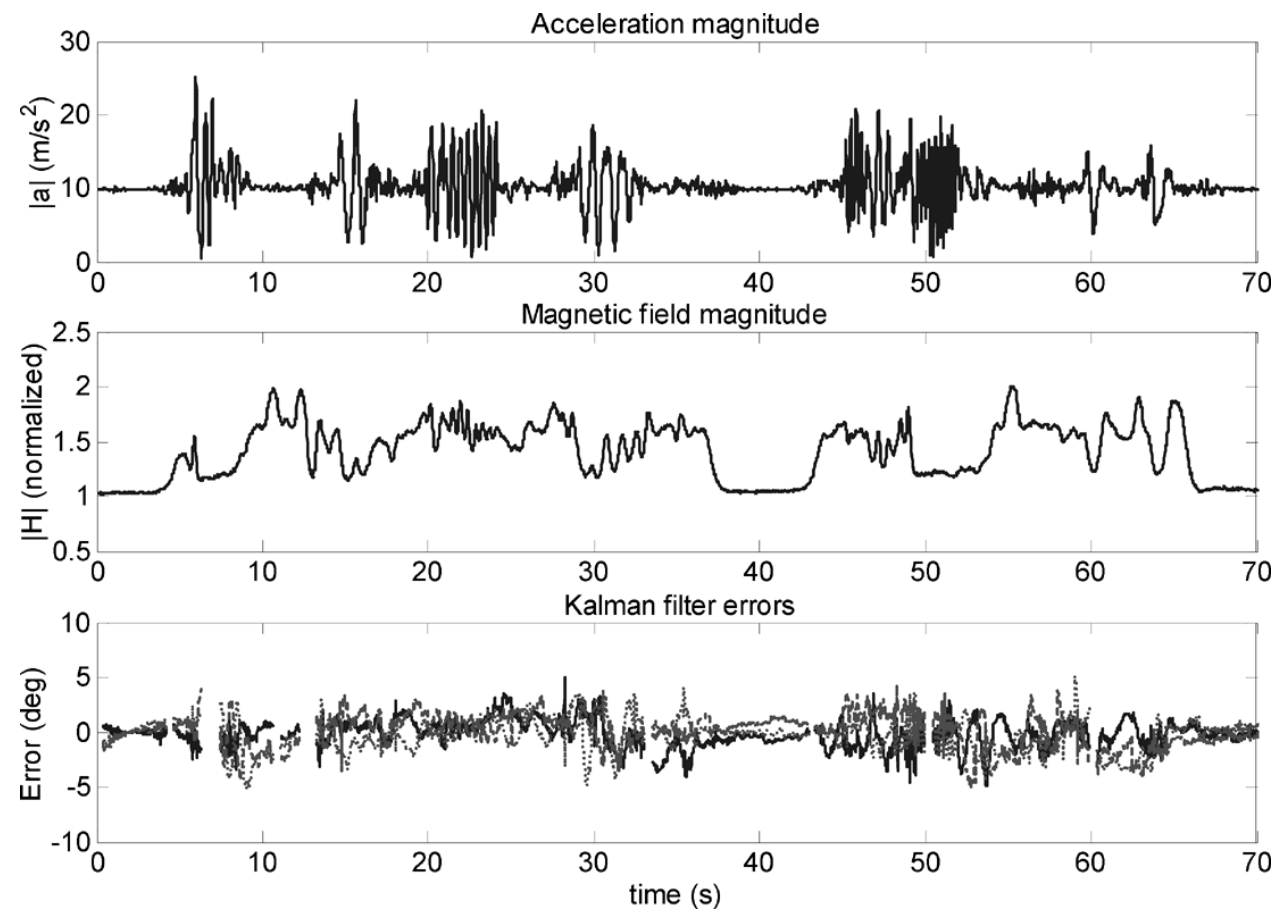

Fig. 11. Results from the sensor signal. Upper: acceleration norm. Upper: magnetic field magnitude. During the movements of the sensor, the magnetic norm is quite variable which is caused by the disturbed magnetic field. Lower: orientation difference between the filter with magnetic disturbance compensation and the optical reference system. Gaps in the data are caused by missed markers from the optical reference system, so no reference orientation could be calculated.

In the case that a sensor module is placed at a fixed distance near ferromagnetic materials, like on prostheses or on tools, the magnetic sensors should be calibrated under these conditions. Soft and hard iron effects as described in [32] should then be taken into account. More ferromagnetic materials in the measurement volume will decrease the filter performance.

The accuracy could also decrease if the disturbance has the same low bandwidth as the gyroscope heading drift or the magnetic field is constantly disturbed. The distinction between heading drift and disturbance is then difficult to make. However, since the magnetic disturbance is modeled as an autoregressive process, these errors are limited. If the sensors are used for example in a moving vehicle, the accelerometer model should be modified. Accelerations of the vehicle will most likely be different from human accelerations solely, resulting in wrong inclination estimates. The acceleration error $\mathbf{a}_{\varepsilon}$ was not modeled as a Kalman state since this error hardly influences the inclination estimate. The estimation of the acceleration a by using the signal prediction model showed stable and accurate results. If a more accurate estimate of the acceleration $\mathbf{a}$ is desired, it could be taken into account in the Kalman filter.

The proposed model and Kalman filter can be applied to any combination of inertial and magnetic sensors. In principle, only the specifications of the sensors have to be known, like noise and drift. As MEMS techniques improve, the next generation of gyroscopes will suffer less from drift [33]-[36]. This means that the accelerometer and magnetometer filter weights can be reduced, resulting in less interference problems. The temperature sensor was only used as a global indication of the stability of the temperature inside the whole sensor module. For accurate temperature drift compensation, a temperature sensor should be mounted directly on the gyroscopes and accelerometers and a temperature model should be available.

In conclusion, the proposed Kalman filter implementation shows accurate and drift free 3-D orientation estimates with the capability to correct for magnetic interferences.

\section{REFERENCES}

[1] M. Munneke, Z. de Jong, A. H. Zwinderman, G. J. Tijhuis, J. M. Hazes, and T. P. V. Vlieland, "The value of a continuous ambulatory activity monitor to quantify the amount and intensity of daily activity in patients with rheumatoid arthritis," J. Rheumatol., vol. 28, no. 4, pp. 745-50, 2001.

[2] H. J. Busser, J. Ott, R. C. van Lummel, M. Uiterwaal, and R. Blank, "Ambulatory monitoring of children's activity," Med. Eng. Phys., vol. 19, no. 5, pp. 440-5, 1997.

[3] M. J. Mathie, A. C. F. Coster, N. H. Lovell, and B. G. Celler, "Detection of daily physical activities using a triaxial accelerometer," Med. Biol. Eng. Comput., vol. 41, no. 03, pp. 296-301, 2003.

[4] C. T. M. Baten, H. van Moerkerk, I. I. Kingma, J. Van Dieën, T. Dolan, P. H. Veltink, and H. J. Hermens, "Ambulatory low back load exposure estimation," presented at the 14th Tri-Annual Congress IEA/ 44th Ann. Meeting HFES, San Diego, CA, 2000.

[5] H. J. Busser, W. de Korte, E. Glerum, and R. van Lummel, "Method for objective assessment of physical work load at the workplace," $E r$ gonomics, vol. 41, no. 10, pp. 1519-1526, 1998.

[6] C. V. C. Bouten, K. T. M. Koekkoek, M. Verduin, R. Kodde, and J. D. Janssen, "A triaxial accelerometer and portable processing unit for the assessment of daily physical activity," IEEE Trans. Biomed. Eng., vol. 44, no. 3, pp. 136-147, Mar. 1997.

[7] M. Uiterwaal, E. B. Glerum, H. J. Busser, and R. C. van Lummel, "Ambulatory monitoring of physical activity in working situations, a validation study," J. Med. Eng. Tech., vol. 22, no. 4, pp. 168-72, 1998.

[8] P. H. Veltink, "Sensory feedback in artificial control of human mobility," Tech. Health Care, vol. 7, no. 6, pp. 383-391, 1999.

[9] P. H. Veltink, P. Slycke, J. Hemssems, R. Buschman, G. Bultstra, and H. Hermens, "Three dimensional inertial sensing of foot movements for automatic tuning of a two-channel implantable drop-foot stimulator," Med. Eng. Phys., vol. 25, no. 1, pp. 21-8, 2003. 
[10] R. E. Mayagoitia, A. V. Nene, and P. H. Veltink, "Accelerometer and rate gyroscope measurement of kinematics: An inexpensive alternative to optical motion analysis systems," J. Biomechanics, vol. 35, no. 4, pp. $537-542,2002$.

[11] L. Williamson and B. J. Andrews, "Detecting absolute human knee angle and angular velocity using accelerometers and rate gyroscopes," Med. Biol. Eng. Comput., vol. 39, no. 03, pp. 294-302, 2003.

[12] E. B. Bachmann, "Inertial and magnetic tracking of limb segment orientation for inserting humans into synthetic environments," Ph.D. dissertation, Naval Postgrad. School, Monterey, CA, 2000.

[13] P. Jonker, S. Persa, J. Caarls, F. Jong de, and I. Lagendijk, "Philosophies and technologies for ambient aware devices in wearable computing grids," Comput. Commun., no. 26, pp. 1145-1158, 2003.

[14] E. Foxlin, "Inertial head-tracker sensor fusion by a complementary separate-bias Kalman filter," in Proc. VRAIS, 1996, pp. 185-194.

[15] D. Giansanti, V. Macellari, G. Maccioni, and A. Cappozzo, "Is it feasible to reconstruct body segment 3-D position and orientation using accelerometric data?," IEEE Trans. Biomed. Eng., vol. 50, no. 4, pp. 476-83, Apr. 2003.

[16] H. J. Luinge and P. H. Veltink, "Inclination measurement of human movement using a 3-D accelerometer with autocalibration," IEEE Trans. Neural Syst. Rehabil. Eng., vol. 12, no. 1, pp. 112-121, Mar. 2004.

[17] G.-Å. Hansson, P. Asterland, N.-G. Holmer, and S. Skerfving, "Validity and reliability of triaxial accelerometers for inclinometry in posture analysis," Med. Biol. Eng. Comp., vol. 39, no. 04, pp. 415-413, 2001.

[18] E. Bernmark and C. Wiktorin, "A triaxial accelerometer for measuring arm movements," Appl. Ergonomics, vol. 33, no. 6, pp. 541-547, 2002.

[19] H. J. Luinge, P. H. Veltink, and C. T. Baten, "Estimating orientation with gyroscopes and accelerometers," Technol. Health Care, vol. 7, no. 6, pp. $455-9,1999$.

[20] M. Haid and J. Breitenbach, "Low cost inertial orientation tracking with Kalman filter," Appl. Math. Comp., vol. 153, no. 2, pp. 567-575, 2003.

[21] B. Kemp, A. J. Janssen, and B. van der Kamp, "Body position can be monitored in 3-D using miniature accelerometers and earth-magnetic field sensors," Electroencephalogr. Clin. Neurophysiol., vol. 109, no. 6, pp. 484-8, 1998.

[22] J. L. Marins, X. Yun, E. R. Bachmann, R. B. McGhee, and M. J. Zyda, "An extended Kalman filter for quaternion-based orientation estimation using MARG sensors," in IEEE/RSJ Int. Conf. Intelligent Robots Syst., vol. 4, 2001, pp. 2003-2011.

[23] H. J. Luinge, "Inertial sensing of human movement," Ph.D. dissertation, University of Twente, Enschede, The Netherlands, 2002.

[24] R. G. Brown and P. Y. C. Hwang, Introduction to Random Signals and Applied Kalman Filtering, 3rd ed. New York: Wiley, 1997.

[25] J. E. Bortz, "A new mathematical formulation for strapdown inertial navigation," IEEE Trans. Aerosp. Electron. Syst., vol. 7, pp. 61-66, 1971.

[26] National Geomagnetism Program. Geomagnetism, P.N. [Online]. Available: http://geomag.usgs.gov

[27] MT9-A Datasheet. Xsens. [Online]. Available: http://www.xsens.com

[28] F. Ferraris, U. Grimalde, and M. Parvis, "Procedure for effortless in-field calibration of three axis rate gyros and accelerometers," Sens. Mater., vol. 7, no. 5, p. 311, 1995

[29] H. J. Luinge and P. H. Veltink, "Measuring orientation of human body segments using miniature gyroscopes and accelerometers," Med. Biol. Eng. Comp., to be published.

[30] Y. Ehara, H. Fujimoto, S. Miyazaki, M. Mochimaru, S. Tanaka, and S. Yamamoto, "Comparison of the performance of 3-D camera systems II," Gait Posture, vol. 5, pp. 251-255, 1997.

[31] C. T. M. Baten, H. Luinge, and H. Moerkerk van, "Estimating body segment body orientation applying inertial sensing," presented at the 6th Int. Symp. 3-D Analysis Human Movement, Cape Town, South Africa, 2000.

[32] M. J. Caruso, "Application of magnetoresistive sensors in navigation systems," Sens. Actuators, 1997.

[33] J. Soderkvist, "Micromachined gyroscopes," Sens. Actuators A, Phys., vol. 43, pp. 65-71, 1994.

[34] S. E. Alper and T. Akin, "A symmetric surface micromachined gyroscope with decoupled oscillation modes," Sens Actuators A, Phys., vol. 97-8, pp. 347-358, 2002.

[35] H. K. Xie and G. K. Fedder, "Integrated microelectromechanical gyroscopes," J. Aerospace Eng., vol. 16, no. 2, pp. 65-75, 2003.

[36] iMEMS Gyroscopes Data Sheets (ADXRS150 \& ADXRS300), Analog Devices, 2003.

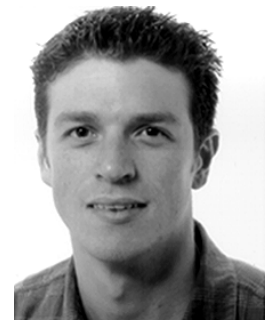

Daniel Roetenberg received the M.Sc. degree in electrical engineering from the University of Twente, Enschede, The Netherlands, in 2001, where he is currently working toward the Ph.D. degree on the topic of inertial and magnetic sensing of human movement.

He was with the Roessingh Research and Development, Enschede, The Netherlands, where he worked on EMG signal processing.

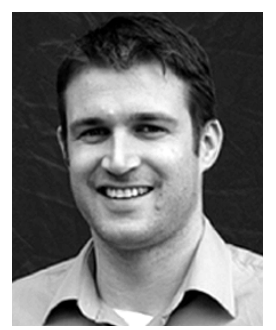

Henk J. Luinge received the M.Sc. and Ph.D. degrees in mechanical engineering from the University of Twente, Enschede, The Netherlands, in 1998 and 2002, respectively. His Ph.D. research was on the topic of inertial sensing of human movement.

After working at Xsens Technologies B.V., Enschede, The Netherlands, he worked as a postdoctoral fellowship at the Rehabilitation Institute of Chicago, Chicago, IL, and the Ecole Nationale Vétérinaire d'Alfort, France. He is currently with Xsens Motion Technologies, Enschede, The Netherlands.

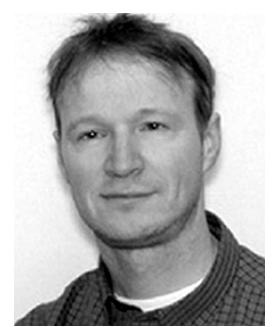

Chris T. M. Baten received the M.Sc. degree in electrical/biomedical engineering from the University of Twente, Enschede, The Netherlands, in 1990.

$\mathrm{He}$ is currently with Roessingh Research and Development, Enschede, The Netherlands, as a Senior Researcher. His research interests include ambulatory monitoring of 3-D motion and internal mechanical joint load exposure monitoring and surface EMG. He is currently National Coordinator of the FreeMotion project aiming at a complete and accurate portable gait laboratory with only wearable sensors. He also has been national coordinator of the preceding Amber and AmbuLab projects on ambulatory back load exposure monitoring and participant in numerous other studies on wearable sensing in human motion analysis.

Mr. Baten is a member of the council of the 3-D analysis of human movement technical group of the International Society of Biomechanics.

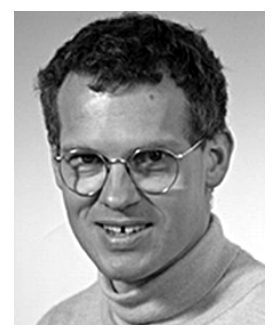

Peter H. Veltink (S' $85-M^{\prime}$ ' 88) was born in Groenlo, The Netherlands, in 1960. He received the M.Sc. and Ph.D. degrees in electrical engineering from the University of Twente, Enschede, The Netherlands, in 1984 and 1988, respectively, His Ph.D. degree research was in the area of electrical nerve stimulation.

Currently, he is a Professor of technology for the restoration of human function at the Institute for Biomedical Technology (BMTI), University of Twente, and performs research in the area of artificial motor control and ambulatory sensory systems with applications to rehabilitation medicine. He has been the Scientific Coordinator of three European Union (EU) research training networks and is and has been involved in various projects financed by the EU, the Dutch ministry of Economic Affairs, and the Dutch Foundation for Technical Sciences STW. He performed sabbaticals at Case Western Reserve University, Cleveland, $\mathrm{OH}$, in 1989 and at the Center for Sensory Motor Interaction at Aalborg University, Aalborg, Denmark, in 1997.

Dr. Veltink was the Treasurer of the International Functional Electrical Stimulation Society (IFESS) from 1996 to 2001. He received the Royal Shell Stimulating Prize for his contribution to the rehabilitation engineering field in 1997. 